\title{
Diatomeas (Bacillariophyceae) en humedales de altura de LA Provincia de Catamarca (Argentina). III
}

\author{
NORA I. MAIDANA ${ }^{1}$ y CLAUDIA T. SEELIGMANN ${ }^{2}$
}

\begin{abstract}
Summary: Diatoms (Bacillariophyceae) in high-altitude wetlands of Catamarca Province (Argentina). III. Within the framework of the research on high mountain wetlands conducted since 1998, we have already studied 24 waterbodies in this province. In order to expand our knowledge on diatom flora in these high mountain environments, in this opportunity we analyze another 12 wetlands located between $25^{\circ} 34^{\prime} 05^{\prime \prime}-$ $27^{\circ} 39^{\prime} 23^{\prime \prime} \mathrm{S}$, over $3000 \mathrm{~m}$ asl. Diatoms were found in all samples, except in Laguna Azul, with almost white water by the large amount of suspended solids. In relation to salinity, waters were from fresh to hypersaline, with a predominance of sodium and chloride ions. We identify, excluding Fragilariaceae, 42 genera with 123 infrageneric taxa, 29 of which are new records for the province of Catamarca and, 4 for Argentina and we propose a new species (Anomoeoneis punae sp nov.). The majority of the species belong to Navicula (17), Nitzschia (13), Halamphora (12), Surirella (8), and Anomoeoneis (7). There were not found species common to all samples and only six appeared in more than $50 \%$ of the waterbodies. A high percentage $(54 \%)$ of rare species, found only in one or two of the samples, was obtained.
\end{abstract}

Key words: Diatoms, wetlands, Andes, Catamarca, Argentina.

\begin{abstract}
Resumen: En el marco de la investigación de humedales de alta montaña que realizamos desde 1998, hemos estudiado ya 24 cuerpos de agua de esta provincia. Con el objetivo de ampliar los conocimientos de la flora diatomológica en ambientes de alta montaña, en esta oportunidad analizamos otros 12 humedales ubicados entre los $25^{\circ} 34^{\prime} 05^{\prime \prime}-27^{\circ} 39^{\prime} 23^{\prime \prime} \mathrm{S}$, a más de $3000 \mathrm{~m}$ snm. Encontramos diatomeas en todas las muestras, excepto en la de la laguna Azul, que es casi blanca por la gran cantidad de sólidos en suspensión. En relación a la salinidad, las aguas fueron desde dulces hasta hipersalinas, con predominio de los iones sodio y cloruro. Identificamos, excluyendo las Fragilariaceae, 42 géneros con 123 taxones infragenéricos, 29 de los cuales son nuevos registros para la provincia de Catamarca, 4 para Argentina y proponemos una nueva especie (Anomoeoneis punae sp nov.). La mayoría de las especies pertenecen a los géneros Navicula (17), Nitzschia (13), Halamphora (12), Surirella (8) y Anomoeoneis (7). No se encontraron especies comunes a todas las muestras y sólo seis tuvieron una frecuencia de ocurrencia mayor al $50 \%$. Se obtuvieron altos porcentajes (54 \%) de especies raras, halladas solo en una o dos de las muestras.
\end{abstract}

Palabras clave: Diatomeas, humedales, Andes, Catamarca, Argentina.

\section{INTRODUCCIÓN}

Durante las últimas décadas se ha incrementado el interés en el estudio de las Bacillariophyceae en humedales de alta montaña de América del Sur (Rumrich et al., 2000; Morales et al., 2007; Álvarez

${ }^{1}$ Dpto. de Biodiversidad y Biología Experimental, Facultad de Cs. Exactas y Naturales, Universidad de Buenos Aires. IBBEA (UBA-CONICET). C. Universitaria, 1428, CABA, Argentina.nim@bg.fcen.uba.ar.

${ }^{2}$ Dpto. de Biología, Facultad de Cs. Naturales e Inst. M. Lillo. M. Lillo 205, 4000, S. M. de Tucumán, Argentina.
Blanco et al. 2011; Blanco et al., 2013) ya que son las algas más abundantes y frecuentes en este tipo de hábitats y las menos estudiadas. Por otra parte, estos ambientes son considerados excelentes escenarios para la reconstrucción, seguimiento y la anticipación de los efectos de cambios globales sobre los ecosistemas.

En Argentina, los numerosos y diversos cuerpos de agua en alta montaña se hallan en los Andes Centrales, que abarcan la región Norte de Argentina y Chile, Oeste de Bolivia y Sur de Perú. Esta región se caracteriza por el predominio de cuencas endorreicas con diferentes tipos de humedales bajo 
un clima árido. La región del Altiplano de la Puna tiene una altitud media de $3700 \mathrm{~m}$ snm y el nivel de base local está hidrológicamente aislado. Este aislamiento ocurre donde el poder de incisión del sistema de drenaje regional se ha reducido de modo importante por medio de una combinación de la disminución de las precipitaciones (menos de 200 $\mathrm{mm} / \mathrm{año}$ ) relacionadas con el clima regional y la orografía local y por afloramientos del basamento resistentes a la erosión (Alonso et al., 2006). Por otra parte, debido a su altura, la Puna se caracteriza por amplitud térmica diaria y radiación solar altas, por lo que la biota que se desarrolla se encuentra sometida a condiciones extremas. De hecho, las redes tróficas son relativamente simples, los consumidores secundarios están representados en su mayor parte por aves y, en general, no hay peces debido a la alta conductividad de las aguas (Caziani \& Derlindati, 2000). La mayor parte de los humedales de altura que hemos estudiado hasta el momento en las provincias de Jujuy y Catamarca son oligosalinos a hipersalinos debido al aporte de sales procedentes de fluidos hidrotermales asociados a fallas y el lavado de rocas volcánicas. Ejemplo de ello es la laguna Diamante que recibe importantes aportes por arrastre de sales de las laderas del volcán Galán. Esta intensa actividad volcánica que caracteriza el área ha favorecido el enriquecimiento en fosfatos y sulfatos de los cuerpos de agua, los cuales poseen una ficoflora dentro de la cual Bacillariophyceae es el grupo mejor representado (Álvarez-Blanco et al., 2011).

A partir del interés manifestado por el Grupo Conservación Flamencos Altoandinos (GCFA) de Argentina, Bolivia y Perú, se iniciaron censos simultáneos de flamencos de los países intervinientes y los muestreos de fito y zooplancton con el fin de determinar la disponibilidad y calidad de alimento para las aves.

Dentro del marco de investigación de humedales de alta montaña con esta contribución son 36 los ambientes de alta montaña estudiados en Catamarca.

\section{Materiales y Métodos}

En el marco del Censo Internacional de Aves Altoandinas del GCFA, realizado en enero de 2005, se muestrearon 12 humedales de la provincia de
Catamarca situados entre los $25^{\circ} 34^{\prime} 05^{\prime \prime}-27^{\circ} 39^{\prime} 23^{\prime \prime}$ S. De la zona litoral de cada uno de ellos, se obtuvo una muestra de agua con red de plancton de $20 \mu \mathrm{m}$ de apertura de malla. La ubicación y los datos de profundidad, oxígeno disuelto (OD), conductividad y salinidad de cada uno se detallan en la Fig. 1 y Tabla 1. Los humedales fueron clasificados en cuanto a su salinidad según Cowardin et al. (1979). El material fue recolectado y fijado in situ con formaldehído al $4 \%$ e incorporado al Herbario de la Fundación Miguel Lillo bajo la denominación LIL 26.100 a LIL 26.111 y corresponden a: Dpto. Antofagasta de la Sierra: Laguna Grande (26.100); Laguna Cabi (26.101); Laguna Carachi Pampa (26.102); Laguna Diamante (26.103); Laguna Baya (26.104); Laguna Salitre (26.105); Laguna Purulla (26.106); Laguna Caro (26.107). Dpto. Tinogasta: Vega Laguna Negra (26.108); Laguna Azul (26.109); Vega Laguna La Coipa (26.110); Vega Ojo de las Losas (26.111).

Para la eliminación de la materia orgánica se utilizó peróxido de hidrógeno (30\%) y calor (Battarbee, 1986). Con el material ya procesado se confeccionaron preparados permanentes utilizando Naphrax ${ }^{\circledR}$ como medio de montaje. Los especímenes fueron examinados con un microscopio Reichert Polivar con contraste de fase interferencial, usando objetivos planapocromáticos de 100x. Las observaciones con microscopio electrónico de barrido (MEB) se realizaron con un equipo Carl Zeiss SUPRA 40, del Centro de Microscopías Avanzadas de la Facultad de Ciencias Exactas y Naturales, Universidad de Buenos Aires.

La identificación taxonómica se basó, en general, en las monografías de Hartley (1996), Metzeltin \& Lange-Bertalot (1998, 2007), Metzeltin et al. (2005), Patrick (1961), Patrick \& Reimer (1966, 1975), Rumrich et al. (2000), Schmidt et al. (1874-1959), Simonsen (1987) Krammer \& Lange-Bertalot (1986, 1988, 1991, 2004) y obras específicas de autores varios que se mencionan en cada caso. Los requerimientos ecológicos de las distintas especies fueron tomados de De Wolf (1982), Van Dam et al. (1994), Lowe (1974) y de la literatura consultada en cada caso. Para la distribución geográfica en Argentina se consultó a Seeligmann \& Maidana (2003), Maidana \& Seeligmann (2006), Seeligmann et al. (2008), Maidana et al. (2009) y Vouilloud (2003). Los taxones identificados en las muestras analizadas 


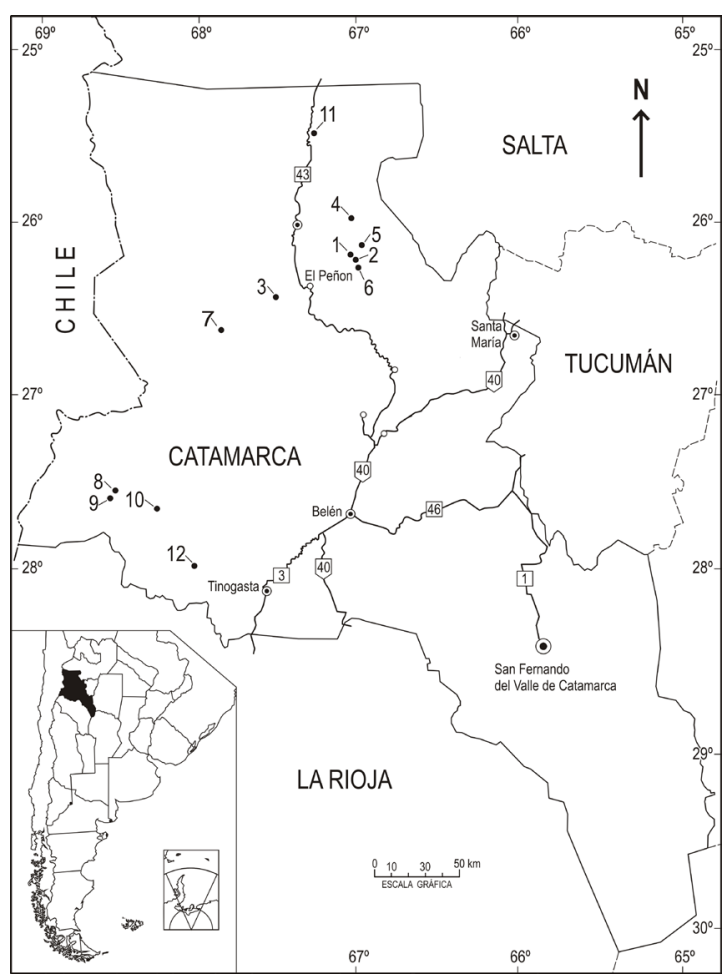

Fig. 1: Ubicación de los 12 humedales muestreados: 1: Laguna Grande, 2: Laguna Cabi, 3: Laguna Carachi Pampa, 4: Laguna Diamante, 5: Laguna Baya, 6: Laguna Salitre, 7: Laguna Purulla, 8: Vega de Laguna Negra, 9: Laguna Azul, 10: Vega de Laguna La Coipa, 11: Laguna Caro, 12 Vega de Ojo de las Losas.

figuran en el Apéndice. Con el fin de examinar la relación entre número de especies y la salinidad, se efectuó un análisis de correlación de Pearson $(\mathrm{p}<0,05, \mathrm{p}<0,01)$ mediante el programa estadístico SPSS (versión 10,0) para Windows.

Dado que la Familia Fragilariaceae presentó una notable riqueza de especies y una gran variabilidad morfológica en los cuerpos de agua estudiados, las especies de esta Familia serán objeto de una publicación aparte.

\section{Resultados y Discusión}

La mayoría de los humedales estudiados son someros (3-30 $\mathrm{cm}$ de profundidad) y, según la clasificación de Cowardin et al. (1979), sus aguas van desde dulces a hipersalinas, con un rango de salinidad comprendido entre 0,41 a 73,95 $\mathrm{g} \mathrm{l}^{-1}$ (Tabla 1). Los datos químicos proporcionados por el GCFA (inéditos), mostraron que las aguas de las lagunas Baya, Carachi Pampa, Caro, Diamante, Grande y Purulla tuvieron en general altas concentraciones de sodio (8915-52.662 $\mathrm{mg} \mathrm{l}^{-1}$ ) y cloruros (8717-58.728 $\mathrm{mg}^{1^{-1}}$; que, además, el magnesio tuvo valores muy elevados en la laguna Purulla (4738 $\mathrm{mg} \mathrm{l}^{-1}$ ) y los sulfatos en las lagunas Carachi Pampa (35.112 mg $\left.\mathrm{l}^{-1}\right)$, Diamante (7250 $\left.\mathrm{mg} \mathrm{l}^{-1}\right)$ y Baya $\left(6700 \mathrm{mg} \mathrm{l}^{-1}\right)$.

Excepto en la laguna Azul donde no se hallaron diatomeas, en los restantes humedales se identificaron 42 géneros con 123 especies y variedades. La mayoría de las especies identificadas pertenece a los géneros Navicula Bory (17), Nitzschia Hassall (13) Halamphora Lepkov (12), Surirella Turpin (8) y Anomoeoneis Pfitzer (7). Veintinueve taxones no pudieron ser identificados a nivel específico a partir de la literatura consultada, tanto de la región como de otras partes del mundo. Muchas de estas entidades probablemente sean taxones nuevos para la ciencia.

Las lagunas Salitre y Diamante y las vegas de laguna Negra y Ojo de las Losas tuvieron la mayor riqueza de especies $(39,25,47$ y 38 respectivamente) y las mínimas se registraron en las lagunas Carachi Pampa (11) y Baya (12) (Apéndice). No se encontraron especies comunes a todas las muestras y sólo 6 estuvieron en más del $50 \%$ de las muestras: Navicula lauca Rumrich \& Lange-Bertalot, $N$. parinacota Rumrich \& Lange-Bertalot, Nitzschia halloyi Maidana \& Herbst, Pinnularia microstauron (Ehrenberg) Cleve, Surirella chilensis Janish y Tryblionella hungarica (Grunow) Frenguelli. Un alto porcentaje de especies estuvo presente en una sola muestra (54\%).

Del total de taxones identificados cuatro se registraron por primera vez para el país, 29 especies fueron registradas por primera vez en la provincia de Catamarca y se propone una nueva especie para la ciencia: Anomoeoneis punae sp nov.

Algunas especies nuevas o poco conocidas para la provincia de Catamarca y el país

Adlafia minuscula (Grunow) Lange-Bertalot in Lange-Bertalot \& Genkal (Figs. 2 A y 4 A).

Lange-Bertalot, 2001: 143, Figs. 108: 4-10; 106: 5-8. 
Tabla 1: Parámetros físicos y químicos de los humedales estudiados. Clasificación de los ambientes según grado de salinidad de acuerdo a Cowardin et al. (1979) (dulce: 0,5 $\mathrm{g} \mathrm{I}^{-1}$; oligosalina: 0,5-5 $\mathrm{g} \mathrm{I}^{-1}$; mesosalina: 5-18 $\mathrm{g} \mathrm{l}^{-1}$; polisalina: $18-30 \mathrm{~g} \mathrm{l}^{-1}$, hipersalina $>30 \mathrm{~g} \mathrm{l}^{-1}$. L: laguna; V: vega.

\begin{tabular}{|c|c|c|c|c|c|c|c|c|}
\hline $\mathrm{N}^{0}$ & Humedales & Coordenadas & $\begin{array}{l}\text { Altura } \\
\mathrm{m} \mathrm{snm}\end{array}$ & $\begin{array}{l}\text { Cond. } \\
\mathrm{mS} \mathrm{cm}^{-1}\end{array}$ & $\begin{array}{c}\text { Salinid. } \\
\mathrm{g}^{-1}\end{array}$ & $\begin{array}{c}\text { Clasificación } \\
\text { salinidad }\end{array}$ & $\begin{array}{l}\text { Prof } \\
\mathrm{cm}\end{array}$ & $\begin{array}{c}\mathrm{OD} \\
\mathrm{mg} \mathrm{l}^{-1}\end{array}$ \\
\hline 1 & L. Grande & $26^{\circ} 12^{\prime} 53^{\prime \prime S} 67^{\circ} 04^{\prime} 55^{\prime \prime} \mathrm{W}$ & 4280 & 6,48 & 5 & Oligosalina & 30 & 6,3 \\
\hline 2 & L. Cabi & $26^{\circ} 17^{\prime} 0^{\prime \prime S} 67^{\circ} 04^{\prime} 02^{\prime \prime W}$ & 4241 & 61 & 51,85 & Hipersalina & $\mathrm{s} / \mathrm{d}$ & s/d \\
\hline 3 & L. Carachi Pampa & $26^{\circ} 26^{\prime} 48^{\prime \prime S} 67^{\circ} 30^{\prime} 34^{\prime \prime W}$ & 3014 & 81 & 68,85 & Hipersalina & 56 & 4,1 \\
\hline 4 & L. Diamante & $26^{\circ} 02^{\prime} 08^{\prime} \mathrm{S} 67^{\circ} 01^{\prime} 35^{\prime \prime} \mathrm{W}^{\prime}$ & 4600 & 87 & 73,95 & Hipersalina & 20 & 2,1 \\
\hline 5 & L. Baya & $26^{\circ} 13^{\prime} 57^{\prime \prime S} 66^{\circ} 58^{\prime} 19^{\prime \prime W}$ & 4337 & 54 & 45,9 & Hipersalina & $s / d$ & $s / d$ \\
\hline 6 & L. Salitre & $26^{\circ} 14^{\prime} 51^{\prime \prime S} 66^{\circ} 54^{\prime} 04^{\prime \prime} \mathrm{W}$ & 4248 & 0,51 & 0,41 & Dulce & $s / d$ & $s / d$ \\
\hline 7 & L. Purulla & $26^{\circ} 37^{\prime} 50^{\prime \prime} \mathrm{S} 67^{\circ} 50^{\prime} 47^{\prime \prime} \mathrm{W}$ & 3833 & 59,2 & 50,32 & Hipersalina & 22 & 7,7 \\
\hline 8 & V. Laguna Negra & $27^{\circ} 39^{\prime} 23^{\prime \prime S} 68^{\circ} 33^{\prime} 43^{\prime \prime} \mathrm{W}$ & 4131 & 16,76 & 14,41 & Mesosalina & 47 & 5,7 \\
\hline 9 & L. Azul & $27^{\circ} 34^{\prime} 16^{\prime \prime S} 68^{\circ} 32^{\prime} 11^{\prime \prime} \mathrm{W}$ & 4465 & 2,46 & 2 & Oligosalina & 12 & 7 \\
\hline 10 & V. L Co. La Coipa & $27^{\circ} 38^{\prime} 00^{\prime \prime S} 68^{\circ} 15^{\prime} 01^{\prime \prime} \mathrm{W}$ & 4205 & $s / d$ & $\mathrm{~s} / \mathrm{d}$ & $s / d$ & $\mathrm{~s} / \mathrm{d}$ & $s / d$ \\
\hline 11 & L. Caro & $25^{\circ} 34^{\prime} 05^{\prime \prime S} 67^{\circ} 17^{\prime} 10^{\prime \prime W}$ & 4014 & 80,5 & 68,4 & Hipersalina & 3 & 4 \\
\hline 12 & V. Ojo de las Losas & $26^{\circ} 56^{\prime} 39^{\prime \prime S} 68^{\circ} 08^{\prime} 51^{\prime \prime} \mathrm{W}$ & 4033 & $s / d$ & $\mathrm{~s} / \mathrm{d}$ & $s / d$ & $s / d$ & $s / d$ \\
\hline
\end{tabular}

Valvas-rómbico, elípticas con ápices obtusos, redondeados. Área axial muy angosta y lineal. Área central indistinguible. Rafe filiforme, recto con fisuras terminales curvadas hacia el mismo lado. Estrías no visibles al MO, fuertemente radiales en el centro y convergentes hacia los extremos.

Dimensiones: Eje apical 11,0-14,0 $\mu \mathrm{m}$; eje transapical 3,2-3,7 $\mu \mathrm{m}$; estrías 40-50 en $10 \mu \mathrm{m}$.

\section{Material estudiado: Lagunas Cabi y Salitre.}

Preferencias ecológicas: Según LangeBertalot (2001) es una especie dulceacuícola, predominantemente oligosapróbica pero no necesariamente oligotrófica.

Obs.: Nueva cita para la provincia de Catamarca. Fue mencionada para Antártida por Frenguelli \& Orlando (1958) y las provincias de Córdoba y Buenos Aires como Navicula minuscula Grunow (Vouilloud, 2003).
Amphora affinis Kützing 1844: 107, Fig. 30: 66 (Fig. 2 B y 4 B).

Levkov, 2009: 32; figs. 46: 1-12; 47: 1-9; 59: 14-21; 154: 3.

Valvas semi-lanceoladas, dorsiventrales, con el margen ventral moderadamente cóncavo, ápices angostamente redondeados y débilmente curvados hacia el lado ventral. Área axial angosta, área central con una fascia moderadamente ancha del lado dorsal y ventral, que se amplía hacia el margen. Fisuras del rafe arqueadas. Estrías dorsales radiales, las ventrales radiales en el centro y convergentes hacia los extremos, formadas por dos aréolas.

Dimensiones: Eje apical 38,3-50,0 $\mu \mathrm{m}$, eje transapical $6,3 \mu \mathrm{m}$; estrías dorsales 14 en $10 \mu \mathrm{m}$, ventrales 13 en $10 \mu \mathrm{m}$.

Material estudiado: Laguna Diamante y Vega Ojo de las Losas. 
N. I. Maidana y C. T. Seeligmann - Diatomeas en humedales de Catamarca
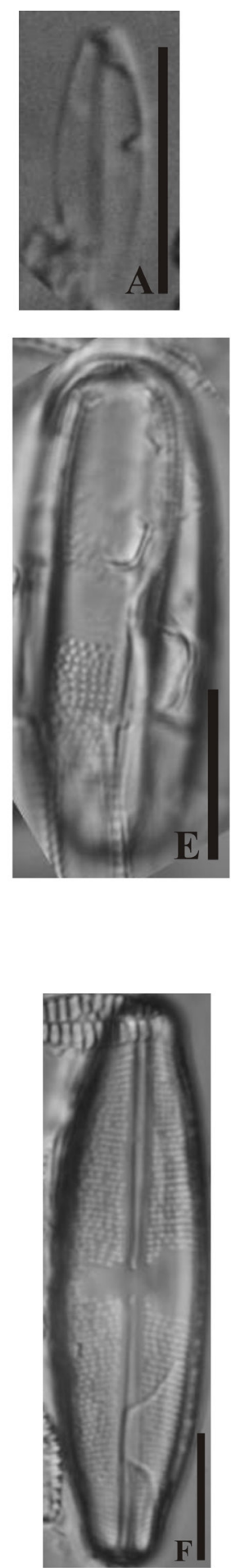
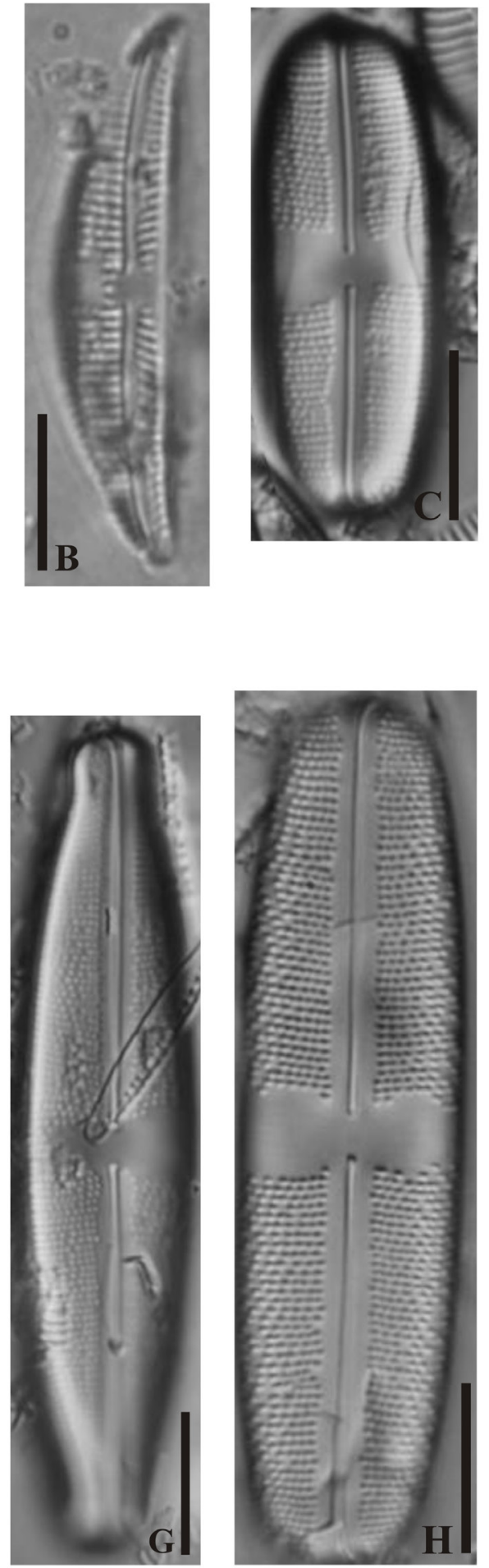
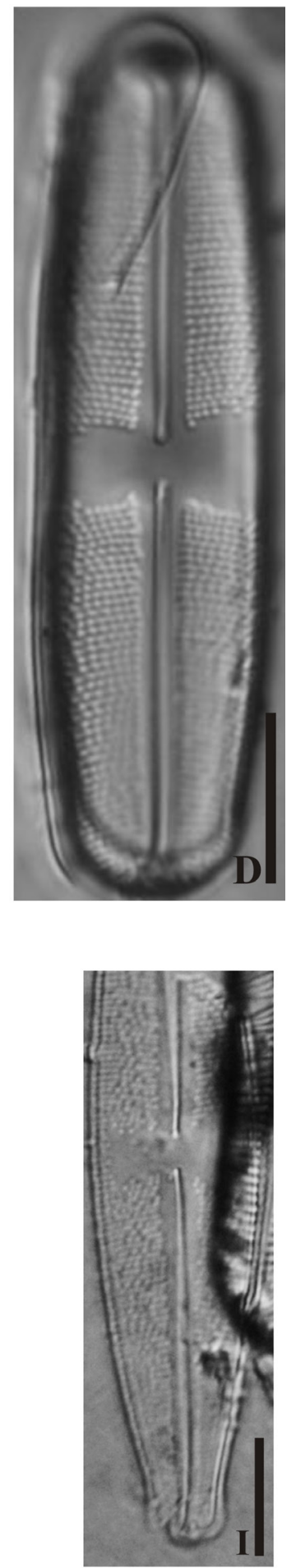

Fig. 2: (microscopio óptico): A: Adlafia minuscula; B: Amphora affinis; C-E; H: Anomoeoneis punae;(C-D; H: Laguna Salitre, Catamarca, Argentina; E: San Pedro de Atacama, Chile); F-G: Anomoeoneis sphaerophora aff. var. angusta Morf. 1; I: A. sphaerophora aff. var. angusta Morf. 2; Escala= $10 \mu \mathrm{m}$. 
Preferencias ecológicas: Especie bentónica, en ambientes circumneutrales a ligeramente alcalinos, meso a ligeramente eutróficos y débilmente salinos (Levkov, 2009). Sin embargo, esta especie fue hallada en un ambiente hipersalino.

Obs.: Citada para la provincia de Córdoba como Amphora ovalis var. affinis (Kütz.) Van Heurck (Vouilloud, 2003). Primera cita para Catamarca. Las dos valvas encontradas en la Laguna Diamante (hipersalina) podrían ser alóctonas, resultado de su transporte, por ejemplo por aves migratorias, desde ambientes menos rigurosos.

Anomoeoneis punae sp nov. (Figs. 2 C-E, H y 4 C-F).

Sinónimos: Anomoeoneis sphaerophora var. angusta Frenguelli sensu Rumrich et al. (2000; lám. 416 , figs. 8 y 9 ).

Navicula novadecipiens Hustedt sensu ServantVildary et al. (2002; http://geologie.mnhn.fr/ diatoms)

Valvae lineares ellipticae, extremis non protractis et apicibus rotundis. Area axialis linearis, 1/4 vel 1/3 $a b$ axe transapicale. Area centralis rectangularis et non aequalis latam fasciam usque ad margines formans. Rhaphe lateralis. Fissurae distales maxime curvatae, sicut falces; fissurae proximales rectae in parum profunda depressione finientes vel ipsam directionem quae distales parum curvatae. Striae uniseriatae, modice radiales in area centrale, dein parallelae et in apicibus convergentes. Areolae plurime parvulae et rotondae, inaequabile dispertitae.

Longitudo: (29,0) 42,2-57,2 $\mu \mathrm{m}$; latitudo $(10,4)$ 12,3-14,5 $\mu \mathrm{m}$; axis apicalis/axis transapicalis 2,94,3; 16-18 striae in $10 \mu \mathrm{m}$, areolae $16-18$ in $10 \mu \mathrm{m}$.

Holotypus: LIL (Herbario Criptogámico) 26.105. In Herbario de la Fundación Miguel Lillo.

Type locality: Laguna Salitre $\left(26^{\circ} 14^{\prime} 51^{\prime \prime} \mathrm{S}\right.$ 6654'04'W), Catamarca, Argentina. 2005.

Localidad tipo: Laguna Salitre (Depto. de Antofagasta de la Sierra, provincia de Catamarca, Argentina)
Valvas lineal-elípticas, con extremos no proyectados y ápices truncadamente redondeados. Área axial lineal, de 1/4 a 1/3 del eje transapical. Área central rectangular y asimétrica formando una fascia ancha y ligeramente asimétrica. Rafe de tipo lateral. En vista externa, las fisuras distales están marcadamente curvadas en forma de hoz y las proximales son rectas y terminan en una depresión poco profunda o están levemente curvadas hacia el mismo lado que las distales. Estrías uniseriadas, levemente radiales en el centro, luego paralelas y convergentes en los ápices. Aréolas, en su mayoría, pequeñas y circulares, irregularmente espaciadas.

Dimensiones: Eje apical $(29,0) 42,2-57,2 \mu \mathrm{m}$; eje transapical $(10,4) 12,3-14,5 \mu \mathrm{m}$; relación eje apical/ eje transapical (EA/ET) 2,9-4,3; estrías 16-18 en 10 $\mu \mathrm{m}$; aréolas 16-18 en $10 \mu \mathrm{m}$.

Material estudiado: Laguna Salitre y Vega Ojo de las Losas.

Preferencias ecológicas: A. punae fue encontrada en aguas con conductividad baja.

Obs.: Los ejemplares observados tanto en Catamarca como en Jujuy (Seeligmann et al., 2008) son idénticos al ejemplar ilustrado como Navicula novadecipiens por Servant-Vildary et al. (2002). Sin embargo, de acuerdo con la descripción original de Hustedt (1966: 659, fig. 1659), esa especie se diferencia de nuestro material y del ilustrado por Servant-Vildary et al. (op. cit.) por la posición excéntrica del rafe, cuyas fisuras distales externas no son largas ni están curvadas.

Además, la especie de Hustedt debería ser estudiada con más detalle y posiblemente deba ser reasignada a otro género ya que, entre otras cosas, no tiene el tipo de areolación característico de Navicula.

Rumrich et al. (2000; lám. 88, figs. 8 y 9) muestran dos valvas muy similares a nuestro material a las que incluyen en Anomoeoneis sphaerophora var. angusta Freng., taxón que se caracteriza, según su autor (Frenguelli, 1934b: 355, Pl. 2, fig. 10), por "la valva lineal lanceolada con extremos bien capitados". Ni nuestro material ni el ilustrado por Rumrich et al. (op. cit.) tienen esas características. 


\section{N. I. Maidana y C. T. Seeligmann - Diatomeas en humedales de Catamarca}

Anomoeoneis atacamensis Frenguelli (1934a: 163, Fig. 2) es semejante en cuanto a la forma general de la valva y del área central pero difiere en la forma de los extremos que son "algo atenuados y subtruncados" y, además, la relación de la longitud del eje apical/eje transapical $(2,76)$ y la densidad de estrías (13 en $10 \mu \mathrm{m})$ son menores que las de $A$. punae. En los preparados de la serie 2813 (Chile), depositada en el Museo de Ciencias Naturales de La Plata, en los cuales Frenguelli reporta la presencia de $A$. atacamensis, no hemos podido encontrar la valva en la que basó la descripción de la especie. Sin embargo, hallamos un frústulo casi completo (Fig. 2, E) con dimensiones diferentes de las de $A$. atacamensis y que coinciden con las de A. punae (34,5 x 10,0 $\mu \mathrm{m}$; estrías 17 en $10 \mu \mathrm{m})$. Esto nos permite extender la distribución geográfica a la localidad de San Pedro de Atacama, en Chile.

Anomoeoneis sphaerophora aff. var. angusta Frenguelli Morfotipo 1 (Fig. 2 F-G).

Frenguelli, 1934b: 355; Fig. 2:10.

Valvas elíptica-lanceoladas con extremos rostrados a rostrado-capitados. Área axial lineal; área central formando una fascia deltoide, asimétrica. Estrías levemente radiales, formadas por pequeñas areolas irregularmente espaciadas, formando líneas irregulares.

Dimensiones: Eje apical 44,8-57,2 $\mu \mathrm{m}$, eje transapical 10,7-13,2 $\mu \mathrm{m}$; estrías 19-21 en $10 \mu \mathrm{m}$.

Material estudiado: Laguna Salitre.

Obs.: Tal como se mencionó anteriormente, la descripción de Frenguelli de Anomoeoneis sphaerophora var. angusta (1934b) dice claramente que los extremos son "bien capitados" mientras que en los de nuestros ejemplares son rostrados a rostrado-capitados. La valva hallada es más angosta, con los extremos rostrados y tiene mayor densidad de estrías. El material ilustrado por Rumrich et al. (2000; lám. 88, figs. 6-9) claramente no corresponde a Anomoeoneis sphaerophora var. angusta Frenguelli.

Anomoeoneis sphaerophora aff. var. angusta Frenguelli Morfotipo 2 (Fig. 2 I).
Frenguelli 1934b: 355; Fig. 2:10

Se diferencia del Morfotipo 1 por sus mayores dimensiones y por tener los extremos netamente rostrados.

Dimensiones: Eje apical $67,3 \mu \mathrm{m}$, eje transapical $15 \mu \mathrm{m}$; estrías 18 en $10 \mu \mathrm{m}$.

Material estudiado: Laguna Diamante.

Obs.: A este morfotipo se aplica las mismas observaciones que al anterior.

Anomoeoneis sphaerophora var. 1 (Fig. 3 A).

Valvas lineal-elípticas, con extremos atenuados, poco diferenciados del cuerpo principal de la valva, y ápices truncadamente redondeados. Área axial irregular, moderadamente ensanchada.

Dimensiones: Eje apical 37,0 $\mu \mathrm{m}$, eje transapical $12,7 \mu \mathrm{m}$; estrías 19 en $10 \mu \mathrm{m}$.

Material estudiado: Vega Ojo de las Losas.

Obs.: Se diferencia de la variedad tipo y de las otras variedades descriptas para la especie por las dimensiones y la forma de los extremos.

Encyonema jemtlandicum var. venezolanum Krammer 1997 (Figs. 3 B y 4 G).

Krammer, 1997: 83, Fig. 14: 1-5; 36:1-3; Rumrich et al. 2000: Fig. 111, fig. 1-4.

Valvas fuertemente dorsiventrales, margen dorsal convexo; ventral, en las formas pequeñas y medianas recto y en las grandes convexo y algo expandido. Extremos no diferenciados, agudos, redondeados. Área axial ventral, algo ensanchada en el centro. Área central no diferenciada de la axial. Estrías suavemente radiales en el centro y convergentes y más densamente dispuestas en los extremos.

Dimensiones: Eje apical 32,6-41,0 $\mu \mathrm{m}$, eje transapical 8,9-11,3 $\mu \mathrm{m}$; relación EA/ET 3.6-3.8; estrías centrales 11-12 en $10 \mu \mathrm{m}$; estrías distales 15 16 en $10 \mu \mathrm{m}$; aréolas $27-30$ en $10 \mu \mathrm{m}$. 
Bol. Soc. Argent. Bot. 50 (4) 2015
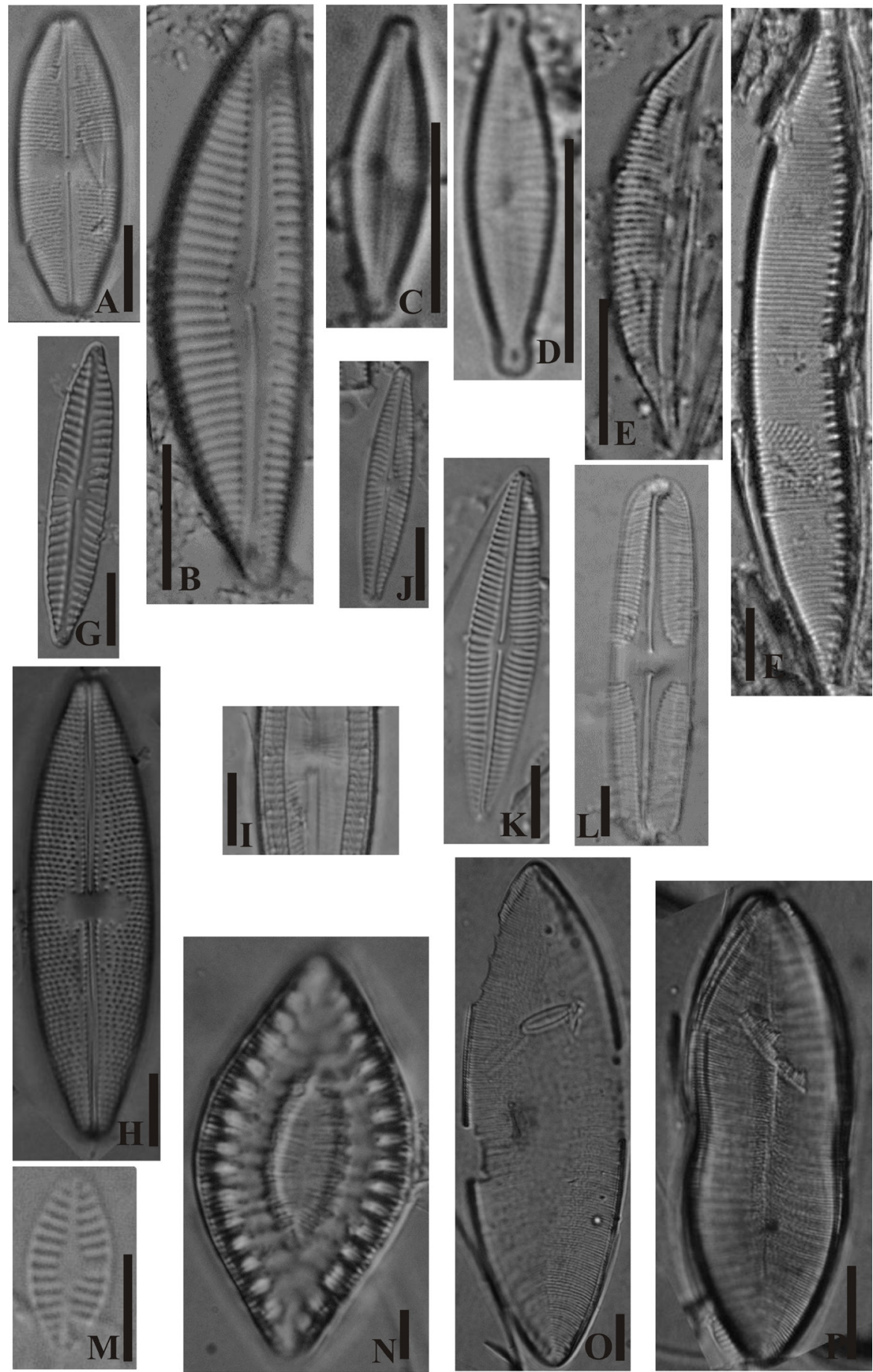

Fig. 3: (microscopio óptico): A: Anomoeoneis sphaerophora var. 1; B: Encyonema jemtlandicum var. venezolanum; C-D: Encyonopsis subfonticola; E: Halamphora aff. luciae; F: Hantzschia hyperborea; G: Navicula aff. pseudogracilis; H-I: Mastogloia aff. patens; J: Navicula sp 1; K: Navicula sp 2; L: Pinnularia aff. boliviana; M: Planothidium aff. minutissimum; N: Surirella chilensis aff. var. tumida; O-P: Surirella moralesi. Escala $=10 \mu \mathrm{m}$. 
N. I. Maidana y C. T. Seeligmann - Diatomeas en humedales de Catamarca
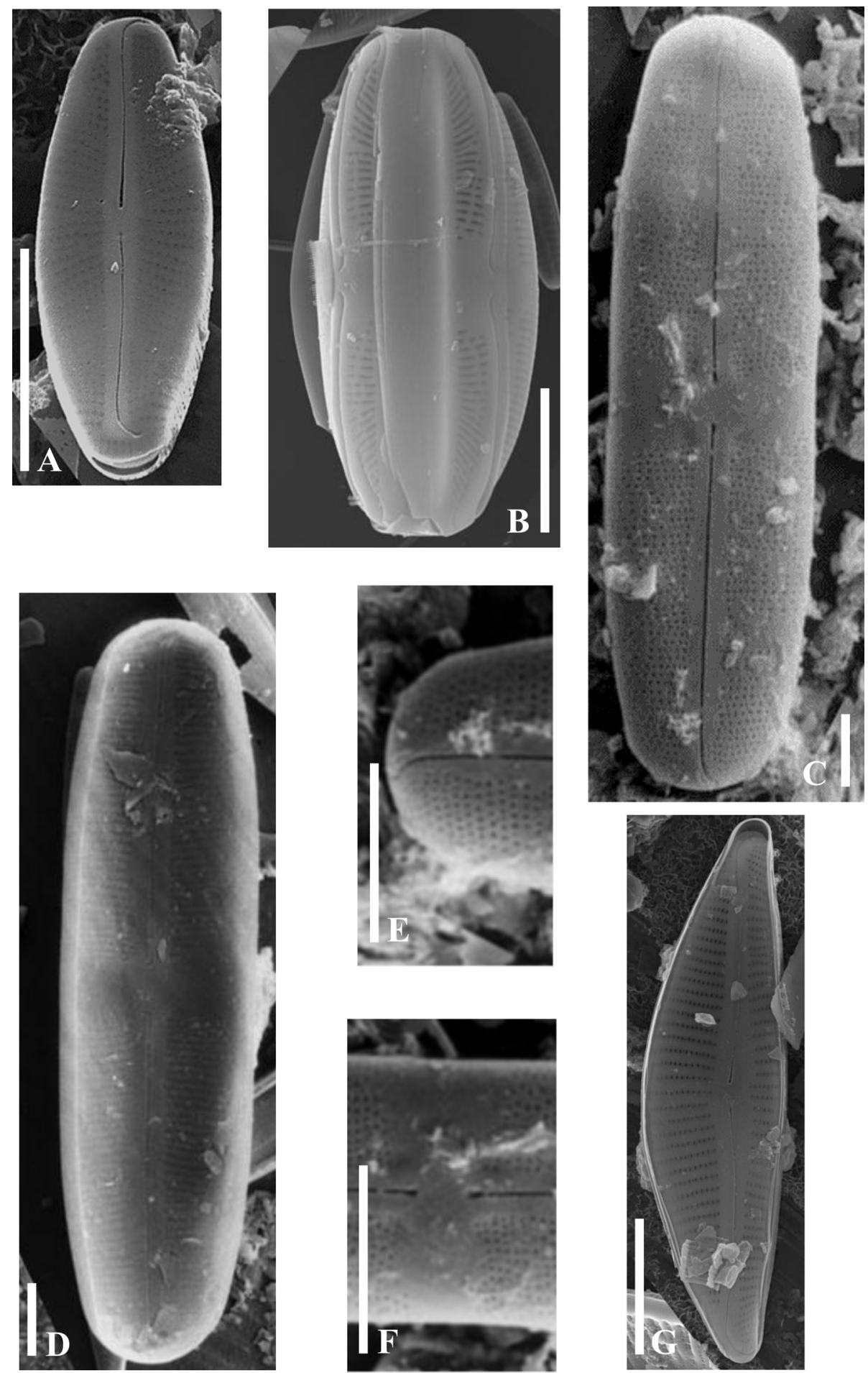

Fig. 4: (microscopio electrónico de barrido): A: Adlafia minuscula; B: Amphora affinis; C-F: Anomoeoneis punae; C. Vista externa; D: Vista interna; E: Detalle de una fisura terminal externa del rafe; F: Fisuras proximales externas del rafe; G: Encyonema jemtlandicum var. venezolanum. Escala $=10 \mu \mathrm{m}$. 


\section{Material estudiado: Laguna Salitre.}

\section{Preferencias ecológicas: Poco conocidas.}

Obs.: Con MEB (Fig. 4 G) se puede observar que las fisuras distales están fuertemente curvadas hacia el margen ventral y las proximales son rectas, con forma de gota. Las estrías, que con MO se ven suavemente radiales, son convergentes en los extremos del lado ventral. Las aréolas son lineoladas. Esta especie fue hallada por Echazú (2012) en humedales del sur de Santa Cruz. Se cita por primera vez para Catamarca.

Encyonopsis subfonticola Krammer 1997 II: 115/152 (Fig. 3 C-D).

Krammer, 1997: 115; Fig. 152: 7-13

Valvas lanceoladas, levemente dorsiventrales, con márgenes dorsales y ventrales diferenciados. Los ejemplares grandes, con extremos angostamente subrostrados y los pequeños, agudos, redondeados. Área axial en ejemplares pequeños muy angosta y en los más grandes algo lanceolada, ensanchada en el centro. El área central ausente. Estrías levemente radiales e igualmente separadas las ventrales y las dorsales.

Dimensiones: Eje apical 15,6-18,3 $\mu \mathrm{m}$, eje transapical 3,7-4,3 $\mu \mathrm{m}$; relación EA/ET 3,6-4,7; estrías $18-20$ en $10 \mu \mathrm{m}$.

Material estudiado: Lagunas Grande y Cabi.

Preferencias ecológicas: Poco conocidas.

Obs.: Nueva cita para el país.

Halamphora chilensis (Hustedt) Levkov 2009 (Fig. $5 \mathrm{~A}$ ).

Levkov, 2009: 177; Figs. 99: 1; 225:1-6

Valvas semi elípticas, con el margen dorsal arqueado y el ventral recto a ligeramente cóncavo. Extremos finamente redondeados y débilmente curvos hacia el lado ventral. Área axial angosta ensanchada cerca del centro. Área central ausente en el lado dorsal de la valva. Fisuras del rafe con los extremos proximales curvados hacia el margen ventral. Estrías uniseriadas y delicadamente areoladas. Estrías dorsales paralelas o débilmente radiales en el centro de la valva y radiadas hacia los extremos. Estrías ventrales radiadas difíciles de ver al MO.

Dimensiones: Eje apical 26, $\mu \mathrm{m}$, eje transapical $6,2 \mu \mathrm{m}$; ancho del frústulo 17,8; estrías dorsales 22-23 en $10 \mu \mathrm{m}$.

Material estudiado: Vega de la Laguna Negra.

Preferencias ecológicas: Especie halófila (Levkov, 2009).

Obs.: Mencionada como Amphora chilensis Hustedt en depósitos fósiles Chile y en diversos lagos salinos actuales de Chile y África (Levcov, 2009). En Argentina está citada para Buenos Aires y Mendoza (Sala et al., 1985). Es una nueva cita para Catamarca.

Halamphora subsalina Levkov 2009 (Fig. 5 B).

Levcov, 2009: 230; Figs. 90: 42-49; 207: 4; 208: $1-7$

Valvas angostas, semi lanceoladas, dorsiventrales con el margen dorsal suavemente arqueado y el ventral ligeramente cóncavo. Extremos ligeramente prolongados y capitados. Área axial angosta, lineal. Área central del lado dorsal ausente y del ventral angosta. Fisuras proximales del rafe dorsalmente curvadas. Estrías dorsales, uniseriadas, formadas por aréolas de forma variable, redondeadas a alargadas transversalmente. Las ventrales formadas por aréolas elongadas, no visibles al MO.

Dimensiones: Eje apical 14,5-15 $\mu \mathrm{m}$, eje transapical $2,5 \mu \mathrm{m}$; estrías dorsales 28 en $10 \mu \mathrm{m}$.

Material estudiado: Vega de laguna Negra.

Preferencias ecológicas: Especie halófila.

Obs.: Solo conocida previamente para la Laguna Blanca (Chile) (Levcov, 2009). Nueva cita para Argentina. 
N. I. Maidana y C. T. Seeligmann - Diatomeas en humedales de Catamarca
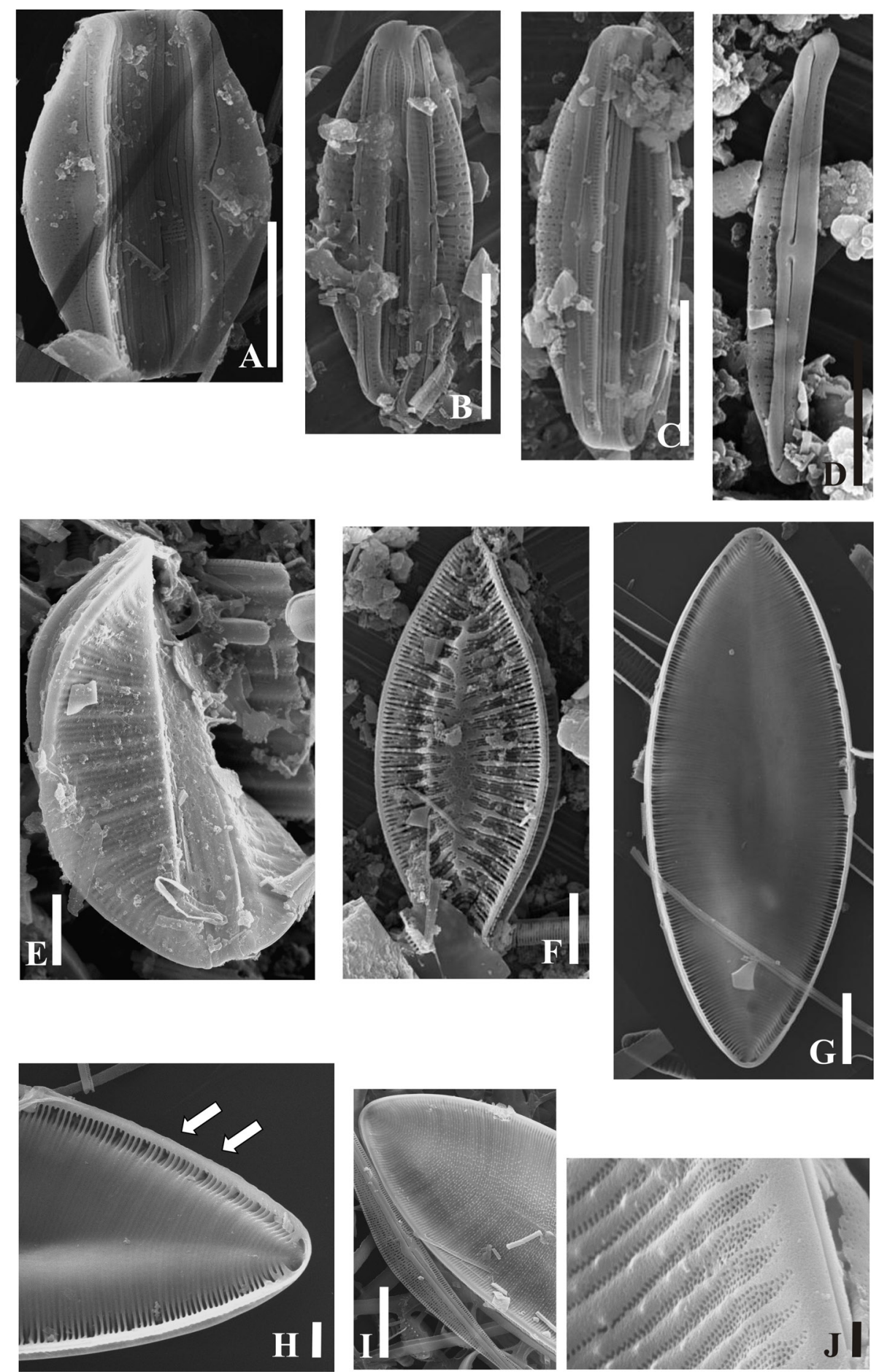

Fig. 5: A: Halamphora chilensis; B: Halamphora subsalina; C: Halamphora aff. luciae; D: Halamphora sp; E: Rhopalodia aff. wetzelli; F: Surirella chilensis aff. var. tumida; G-J: Surirella moralesi; G: Vista interna; H: Detalle mostrando la depresión cada 3-7 fíbulas (flechas); I: Vista externa; J: Vista externa, detalle de las estrías. Escala $=10 \mu \mathrm{m}$ (Figs. A-G; I); = $5 \mu \mathrm{m}$ (Figs. H y J). 
Halamphora aff. luciae (Cholnoky) Levkov 2009 (Figs. 3 E y 5 C).

Levcov, 2009: 203; Figs. 104 (26-36); 242 (5-7)

Valvas semi lanceoladas, con margen dorsal débilmente arqueado y ventral recto o suavemente cóncavo. Extremos diferenciados, capitados y curvados hacia el lado ventral. Área axial muy angosta poco ensanchada en el lado ventral. Área central en el lado dorsal ausente y en el ventral no diferenciable del área axial. Fisuras de rafe poco arqueadas hacia el lado dorsal. Estrías dorsales finamente punteadas, paralelas en el centro y radiales hacia los extremos.

Dimensiones: Eje apical 26,3-30,5 $\mu \mathrm{m}$, eje transapical 3,9-4,8 $\mu \mathrm{m}$; estrías dorsales $18-23$ en $10 \mu \mathrm{m}$.

Material estudiado: Vega de la laguna Negra.

Obs.: No hemos podido observar con MEB vistas internas de las valvas para corroborar la presencia de la costilla longitudinal paralela al rafe, característica de la especie.

\section{Halamphora sp (Fig. 5 D).}

Valvas angostas, semilanceoladas, dorsiventrales, débilmente arqueadas. Margen ventral cóncavo. Extremos ligeramente prolongados y capitados. Área axial angosta. Estrías dorsales con aréolas diminutas y circulares, más laxamente dispuestas hacia el margen dorsal que cerca del esternón; las ventrales formadas por una sola aréola.

Dimensiones: Eje apical 17,4 $\mu \mathrm{m}$; eje transapical $2,5 \mu \mathrm{m}$; estrías dorsales 27 en $10 \mu \mathrm{m}$ y ventrales 40 en $10 \mu \mathrm{m}$.

\section{Material estudiado: Laguna Diamante.}

Obs.: El ejemplar, que solo fue observado con MEB, guarda cierta semejanza con $H$. salinicola Levkov \& Díaz y con $H$. subsalina Levkov. Se diferencia de la primera porque las estrías ventrales están formadas por una sola aréola y de la segunda por la forma de las aréolas del lado dorsal (Levcov, 2009).
Hantzschia hyperborea (Grunow) Lange-Bertalot (Fig. 3 F).

Reichardt, 2008: 17-22; Figs. 1-11 y 11-18.

Valvas dorsiventrales, con el margen dorsal planamente convexo y el ventral suavemente deprimido. Extremos angostos, rostrados. Fíbulas cortas. Estrías centrales algo más distanciadas entre sí que las restantes.

Dimensiones: Eje apical 95,8 $\mu \mathrm{m}$; eje transapical $15,7 \mu \mathrm{m}$; estrías $12-13$ en $10 \mu \mathrm{m}$; fíbulas 5 en $10 \mu \mathrm{m}$.

Material estudiado: Laguna Salitre.

Preferencias ecológicas: Poco conocida.

Obs.: Esta especie ha sido hallada hasta el momento solo en el Altiplano chileno, por encima de los $4000 \mathrm{~m}$ snm (Rümrich et al., 2000). Nueva cita para Argentina.

Mastogloia aff. patens Frenguelli 1936 (Figs. 3 H-I).

Sar et al., 2009: 192; Figs. 17-21 y 5 b.

Valvas elíptico-lanceoladas, extremos subrostrados conápices truncadamenteredondeados. Rafe levemente ondulado, casi recto. Área axial muy angosta, área central transversalmente rectangular, algo asimétrica. Estrías radiales formadas por aréolas gruesas, ordenadas delimitando líneas longitudinales irregularmente onduladas.

Dimensiones: Eje apical 57,7-64,4 $\mu \mathrm{m}$; eje transapical 14,0-17,9 $\mu \mathrm{m}$; estrías $10-12$ en $10 \mu \mathrm{m}$; 10 aréolas en $10 \mu \mathrm{m}$; 8 -9 cámaras partectales en 10 $\mu \mathrm{m}$, de $1,2-1,3 \times 3 \mu \mathrm{m}$.

Material estudiado: Vega de Laguna Negra.

Obs.: El material estudiado se asemeja en la forma general de la valva y del área central pero difiere de $M$. patens en la menor longitud del eje transapical y la mayor densidad de estrías, de areolas y de cámaras partectales. 


\section{N. I. Maidana y C. T. Seeligmann - Diatomeas en humedales de Catamarca}

Navicula aff. pseudogracilis Hustedt 1927 (Fig. 3 G).

Hustedt, 1927: 244, Lám. 8, Fig. 4.

Valvas linear-lanceoladas con extremos obtusamente diferenciados. Área axial angosta, linear, área central muy pequeña, asimétrica, poco diferenciada, delimitada por estrías un poco más cortas. Estrías radiales, robustas, más distanciadas en el centro.

Dimensiones: Eje apical 43,3-44,8 $\mu \mathrm{m}$; eje transapical 7,5-8,0 $\mu \mathrm{m}$; estrías 6-7 en $10 \mu \mathrm{m}$.

\section{Material estudiado: Vega de Laguna Negra.}

Obs.: Las valvas observadas en este trabajo tienen las estrías centrales notablemente separadas pero, a diferencia de N. pseudogracilis, las valvas son más lineales, con el área axial más pequeña y los extremos más capitados. Tanto Rumrich et al. (2000) como Blanco et al. (2012) ilustran ejemplares de $N$. pseudogracilis con las estrías centrales mucho menos distanciadas que las ilustradas por Hustedt en la descripción original de la especie.

\section{Navicula sp 1 (Fig. 3 J).}

Valva elíptico-lanceolada con extremos subrostrados. Área axial angosta, linear, área central orbicular, pequeña y ligeramente asimétrica. Estrías radiales en el centro y convergentes hacia los extremos.

Dimensiones: Eje apical 33,4 $\mu \mathrm{m}$; eje transapical $6,0 \mu \mathrm{m}$; estrías 11 en $10 \mu \mathrm{m}$.

\section{Material estudiado: Vega Ojo de las Losas.}

Obs.: No hemos hallado taxones semejantes en la literatura consultada.

\section{Navicula sp 2 (Fig. 3 K).}

Valva linear-lanceolada con extremos cuneados. Área axial angosta, linear, área central asimétrica, con la estría central más corta sobre uno de los lados y una estría ligeramente más corta a cada lado. Estrías ligeramente radiales en el centro y levemente convergentes en los extremos.

Dimensiones: Eje apical 51,0 $\mu \mathrm{m}$; eje transapical $10,0 \mu \mathrm{m}$; estrías 10 en $10 \mu \mathrm{m}$.

Material estudiado: Laguna Caro.

Obs.: No hemos hallado taxones semejantes en la literatura consultada.

Pinnularia aff. boliviana Blanco et al., 2012 (Fig. $3 \mathrm{~L})$.

Blanco et al., 2012: 15, Figs. 8-10, 34-38.

Valvas lineares con lados paralelos, extremos anchamente redondeados. Rafe filiforme. Área axial que se ensancha desde los extremos hacia la gran área central, romboidal, la que forma una fascia ancha. Estrías radiales en el centro y convergentes en los extremos. Líneas longitudinales ausentes.

Dimensiones: Eje apical $61,0 \mu \mathrm{m}$; eje transapical $13,3 \mu \mathrm{m} ; 12$ estrías en $10 \mu \mathrm{m}$.

Material estudiado: Laguna Cabi.

Obs.: Si bien la forma de la valva es ligeramente diferente, ya que los márgenes no son convexos, ni los ápices son subrostrados, este ejemplar se asemeja a Pinnularia boliviana en el resto de sus características.

Planothidium aff. minutissimum (Krasske) Morales 2006 (Fig. 3 M).

Morales, 2006: 338: Figs. 57-66, 91-96.

Valvas rómbicas-lanceoladas, con ápices obtusos o levemente rostrados. VSR: área axial lanceolada; área central unilateral. Estrías paralelas en la zona media de la valva, levemente convergentes hacia los extremos.

Dimensiones: Eje apical 9,0-10,8 $\mu \mathrm{m}$; eje transapical 4,5-4,7 $\mu \mathrm{m} ; 12-15$ estrías en $10 \mu \mathrm{m}$.

Material estudiado: Vega de la Laguna Negra. 
Obs.: Si bien se asemeja a Planothidium minutissimum en sus dimensiones y en las características de la VSR, consideramos necesario contar con la VCR para definir la especie.

Rhopalodia aff. wetzeli Hustedt (Fig. 5 E).

Hustedt, 1927: 108, Lám. 170, Figs. 1-3.

Frústulo torsionado sobre el eje apical. Valvas dorsiventrales con el lado dorsal arqueado y el ventral plano.

Dimensiones: Eje apical 56,0-88,0 $\mu \mathrm{m}$; eje transapical 15,0-17,5 $\mu \mathrm{m} ; 12$ estrías en $10 \mu \mathrm{m} ; 30$ costillas en $100 \mu \mathrm{m}$.

Material estudiado: Laguna Purulla y Vega de Laguna Negra.

Obs.: Los ejemplares hallados son más pequeños que lo mencionado por Hustedt (70200 x 40-100 $\mu \mathrm{m})$ pero coinciden en la densidad de estrías (10-12 en $10 \mu \mathrm{m})$ y costillas (30 en 100 $\mu \mathrm{m})$.

Surirella chilensis aff. var. tumida Hustedt 1927 (Figs. $3 \mathrm{~N}$ y $5 \mathrm{~F}$ ).

Schmidt et al., 1874-1959; Lám. 362, Fig. 6.

Valvas isopolares, lanceoladas, con extremos cuneados, redondeados, subrostrados. Área axial anchamente elíptica, con una ondulación central.

Dimensiones: Eje apical 45,6-62,2 $\mu \mathrm{m}$; eje transapical 19,3-25,0 $\mu \mathrm{m}$; estrías 19 en $10 \mu \mathrm{m}$ en el centro y 15 en $10 \mu \mathrm{m}$ en los extremos; 25-40 costillas en $100 \mu \mathrm{m}$.

Material estudiado: Lagunas Carachi Pampa y Diamante y Vega Ojo de las Losas.

Obs.: El material estudiado coincide con el aspecto general y la densidad de estrías (18 estrías en $10 \mu \mathrm{m})$ y costillas (30 costillas en 100 $\mu \mathrm{m})$ de esta variedad mencionado por Hustedt (1927) pero sus dimensiones son menores que lo que muestran las fotos del tipo de Husted, en Simonsen (1987) $(79,9 \times 45-48 \mu \mathrm{m})$.
Surirella moralesi S. Blanco, Álvarez-Blanco \& Cejudo-Figueiras 2013 (Fig. 3 O-P y 5 G-J).

Blanco et al., 2013: 22-24, Figs. 44-46, 54-58.

Valvas isopolares, elípticas a lineares-elípticas con extremos cuneados, redondeados. Superficie valvar con una depresión marcada, lanceolada en el área axial la que se hace linear hacia los extremos. Fíbulas débilmente desarrolladas, perpendiculares al eje transapical en el centro y fuertemente convergentes cerca de los extremos. Internamente, el canal rafidiano está sostenido por múltiples fíbulas muy delicadas que se prolongan como costillas hasta el centro de la valva. Cada 3-7 fíbulas se observa una depresión que coincide con lo que se aprecia al MO como canales. (Fig. $5 \mathrm{H}$ ).

Dimensiones: Eje apical 62,6-143,0 $\mu \mathrm{m}$; eje transapical 24,9-38,8 $\mu \mathrm{m}$; 34-40 fíbulas en $100 \mu \mathrm{m}$; 17-20 estrías en $10 \mu \mathrm{m}$.

Material estudiado: Vega Ojo de las Losas.

Preferencias ecológicas: En lagos salinos del Altiplano.

Obs.: Las depresiones arriba mencionadas no fueron observadas por Blanco et al. (1913), si bien se aprecian claramente en su figura 54. Es una nueva cita para Argentina. Por otra parte, encontramos una valva (Fig. 3 P) que se asemeja en todos sus caracteres a $S$. moralesi pero es constricta en su parte media. Sus dimensiones fueron: eje apical 89,0 $\mu \mathrm{m}$; eje transapical 26,21 $\mu \mathrm{m}$; 36 fíbulas en $100 \mu \mathrm{m}$; 18 estrías en $10 \mu \mathrm{m}$.

\section{Conclusiones}

Hasta el momento y teniendo en cuenta este trabajo, analizamos ya 61 cuerpos de agua de alta montaña (Seeligmann \& Maidana, 2003; Maidana \& Seeligmann, 2006; Seeligmann et al., 2008; Maidana et al., 2009) de las provincias de Jujuy y Catamarca (25 y 36 respectivamente) y hemos observado que en esta última se ha encontrado el mayor número de especies exclusivas (212). Del total de taxones identificados en este trabajo (123), 65 de ellos estuvieron presentes en al menos un ambiente 


\section{N. I. Maidana y C. T. Seeligmann - Diatomeas en humedales de Catamarca}

hipersalino y el $21 \%$ no pudieron ser identificadas a nivel de especie. Probablemente algunos de estos taxones sean nuevos para la ciencia. Además, con este aporte se incrementa significativamente el conocimiento de la flora diatomológica existente para Catamarca (29 nuevos registros) y se dan a conocer 4 nuevas citas para el país: Encyonopsis subfonticola Krammer, Halamphora subsalina Levkov (Grunow), Hantzschia hyperborea LangeBertalot y Surirella moralesi Blanco et al.

Algunos autores (Blinn, 2001; ServantVildary, 1978, 1984, Servant-Vildary et al., 2001) postulan que el número de especies presentes en un determinado cuerpo de agua está en relación inversa a la concentración de sales. En este trabajo observamos que los menores valores de riqueza específica ocurrieron tanto en humedales oligosalinos (Laguna Grande) como hipersalinos (lagunas Carachi Pampa, Baya y Purulla). No hallamos una correlación significativa entre la salinidad y el número de especies, lo que, a su vez, coincide con lo que ya mencionamos para el complejo lagunar Vilama en la provincia de Jujuy (Maidana et al., 2009). Es posible que esta discrepancia con lo observado por otros autores pueda ser atribuida a factores tales como la composición iónica y estabilidad en el régimen de agua de los humedales estudiados en cada caso o tipo de muestras analizadas (agua o sedimentos superficiales).

\section{Bibliografía}

ALONSO, R. N., B. BOOKHAGEN, B. CARRAPA, I. COUTLAND, M. HASCHKE, G. E. HILLEY, L. SCHOENBOHN, E. SOBEL, M. R. STRECKER, M. H. TRAUTH \& A. VILLANUEVA. 2006. Tectonics climate and landscape evolution of the Sothern Central Andes: The Argentina Puna Plateau and adjacent regions between 22 and $30^{\circ} \mathrm{S}$ lat. In: ONCKEN, O., G. CHONG, G. FRANZ, P. GIESE, H. GOTZE, et al. (eds.), The Andes-Active Subduction Orogeny; Frontiers in Earth Sciences, pp. 265-283. Springer Verlag, Heidelberg.

ÁLVAREZ-BLANCO I., C. CEJUDO-FIGUEIRAS, I. DE GODOS, R. MUÑOZ \& S. BLANCO. 2011. Las diatomeas de los salares del Altiplano boliviano: singularidades florísticas. Bol. R. Soc. Esp. Hist. Nat. Sec. Biol. 105: 67-82.

BATTARBEE, E. W. 1986. Diatom Analysis. In:
BERGLUND, B. E. (ed.), Handbook of Holocene Palaeoecology and Palaeohydrology, pp. 527-570. J. Wiley \& Sons Ltd., New York.

BLANCO, S., I. ÁlVAREZ-BLANCO, C. CEJUDOFIGUEIRAS, I. DE GODOS, E. BÉCARES, R. MUÑOZ, H. O. GUZMAN, V. A. VARGAS \& R. SOTO. 2013. New diatom taxa from high-altitude Andean saline lakes. Diatom Res. 28: 13-27.

BLINN, D. W. 2001. Diatom community structure along physicochemical gradients in saline lakes. Ecology 74: 1246-1263.

CAZIANI, S. \& E. DERLINDATI. 2000. Abundance and habitat of Andes flamingos in northwestern Argentina. Waterbirds 23: 121-133.

COWARDIN, L. M., V. CARTER, F. C. GOLET \& E. T. LAROE. 1979. Classification of wetlands and deepwater habitats of the United States. US Department of the Interior, US Fish and Wildlife Service, Washington, DC.

DE WOLF, H. 1982. Method of coding of ecological data from diatoms for computer utilization. Mededel. Rijks Geol. Dienst. 36: 95-110.

DÍAZ, C. \& N. I. MAIDANA. 2005. Diatomeas de los salares de Atacama y Punta Negra II región. Chile. Centro de Ecología Aplicada, Santiago.

ECHAZÚ, D. M. 2012. Biodiversidad de diatomeas en humedales del sur de la Provincia de Santa Cruz, Argentina. Tesis doctoral, Universidad Nacional de Buenos Aires.

FRENGUELLI, J. 1934a. Diatomeas del trípoli de San Pedro de Atacama. Revista Chilena Hist. Nat. 38: 159-163.

FRENGUELLI, J. 1934b. Contribución al conocimiento de las diatomeas argentinas. VIII, Diatomeas del Plioceno Superior de las Guayquerías de San Carlos (provincia de Mendoza). Rev. Mus. La Plata 34: 339-371.

FRENGUELLI, J. \& H. A. ORLANDO. 1958. Diatomeas y Silicoflagelados del Sector Antártico Sudamericano. Publ. Inst. Antártico Argentino 5: 1-191.

HARTLEY, B. 1996. An Atlas of British Diatoms. Biopress Limited, Bristol.

HUSTEDT, F. 1927. Fossile Bacillariaceen aus dem Loa- Becken in der Atacama- Wüste, Chile. Arch. Hydrobiol. 18: 224-251.

HUSTEDT, F. 1959-1966. Die Kieselalgen. In: RABENHORST, L. (ed.), Kryptogamen-flora von Deutschland, Osterreich und der Schweiz, 7 (2) 6: 737-845 (1959); 7 (3) 1: 1-160 (1961); 7 (3) 2: 161 348 (1962); 7 (3) 4: 557-816 (1966). Akademische Verlagsgesselsschaft, Greest \& Porting K. G., Leipzig.

KRAMMER, K. 1997. Die cymbelloiden Diatomeen, eine Monographie der weltweit bekannten Taxa. 
Teil 1. Allgemeines und Encyonema Part. Biblioth. Diatomol. 36: 166.

KRAMMER, K. \& H. LANGE-BERTALOT. 1986. Bacillariophyceae 1. Teil: Naviculaceae. In: ETTL, H., J. GERLOFF, H. HEYNIG \& D. MOLLENHAUER (eds.), Süsswasserflora von Mitteleuropa. G. Fischer, Jena.

KRAMMER, K. \& H. LANGE-BERTALOT. 1988. Bacillariophyceae. 2. Teil: Bacillariaceae, Epithemiaceae, Surirellaceae. In: ETTL, H., J. GERLOFF, H. HEYNIG \& D. MOLLENHAUER (eds.), Süsswasserflora von Mitteleuropa. G. Fischer, Jena.

KRAMMER, K. \& H. LANGE-BERTALOT. 1991. Bacillariophyceae. 3. Teil: Centrales, Fragilariaceae, Eunotiaceae. In: ETTL, H., J. GERLOFF, H. HEYNIG \& D. MOLLENHAUER (eds.), Süsswasserflora von Mitteleuropa. G. Fischer, Jena.

KRAMMER, K. \& H. LANGE-BERTALOT. 2004. Bacillariophyceae. 4. Teil: Achnanthaceae Kritische Ergänzungen zu Navicula (Lineolatae) und Gomphonema. In: ETTL, H., J. GERLOFF, H. HEYNIG \& D. MOLLENHAUER (eds.), Süsswasserflora von Mitteleuropa. G. Fischer, Jena.

LANGE-BERTALOT, H. 2001. Diatoms of Europe 2. Navicula sensu stricto. 10 Genera Separated from Navicula sensu lato. Frustulia. A.R.G. Gantner Verlag.

LEVCOV, Z. 2009. Diatoms of Europa 5. Amphora sensu lato. A.R.G. Gantner Verlag K. G. Germany.

LICURSI, M., N. GÓMEZ \& J. DONADELLI. 2010. Ecological optima and tolerances of coastal benthic diatoms in the freshwater-mixohaline zone of the Río de la Plata estuary. Mar. Ecol. Prog. Ser. 418: 105-117

LOWE, R. L. 1974. Environmental requirements and pollution tolerance of freshwater diatoms. National Environmental Research Center. U. S. Environmental Protection Agency, Cincinnati, Ohio.

LUCHINI, L. \& C. A. VERONA. 1972. Catálogo de las diatomeas argentinas. I. Diatomeas de aguas continentales (incluido el Sector Antártico). Comisión de Investigaciones Científicas de la Provincia de Buenos Aires. Monografía $N^{\circ} 2, \mathrm{La}$ Plata.

MAIDANA N. I. \& C. SEELIGMANN. 2006. Diatomeas (Bacillariophyceae) de ambientes acuáticos de altura de la Provincia de Catamarca, Argentina II. Bol. Soc. Argent. Bot. 41: 1-13.

MAIDANA, N. I., C. SEELIGMANN \& M. MORALES. 2009. Bacillariophyceae del Complejo Lagunar Vilama (Jujuy, Argentina). Bol. Soc. Argent. Bot. 44: 257-271.

METZELTIN, D. \& H. LANGE-BERTALOT. 1998. Tropical diatoms of South America I: About
700 predominantly rarely known or new taxa representative of the neotropical flora. Iconogr. Diatomol. 5: 1-695.

METZELTIN, D. \& H. LANGE-BERTALOT. 2007. Tropical diatoms of South America II: Special remarks on biogeographic disjunction. Iconogr. Diatomol. 18: 1-877.

METZELTIN, D., H. LANGE-BERTALOT \& F. GARCÍA-RODRÍGUEZ. 2005. Diatoms of Uruguay; taxonomy-biogeography-diversity. Iconogr. Diatomol. 15: 1-736.

MORALES, E. A. 2006. Small Planothidium Round et Bukhtiyarova (Bacillariophyceae) taxa related to $P$. daui (Foged) Lange-Bertalot from the United States. Diatom Res. 21: 325-342.

MORALES, E. A., M. L. VIS, E. FERNÁNDEZ \& J. P. KOCIOLEK. 2007. Epilithic diatoms (Bacillariophyta) from cloud forest and alpine streams in Bolivia, South America II: A preliminary report on the diatoms from Sorata, Department of La Paz. Acta Nova 3: 680-696.

MÜLLER, O. 1899. Bacillariaceen aus den Natronthälern von El Kab (Ober-Aegypten). Hedwigia 38: 274321.

PATRICK, R. 1961. Diatoms (Bacillariophyceae) from the alimentary tract of Phoenicoparrus jamesi (Sclater). Postilla 49: 43-55.

PATRICK, R. \&. C. W. REIMER. 1966. The diatoms of the United States (exclusive of Alaska and Hawaii). Vol. 1. Acad. Nat. Sci. Philadelphia.

PATRICK, R. \&. C. W. REIMER. 1975. The diatoms of the United States (exclusive of Alaska and Hawaii). Vol 2. Acad. Nat. Sci. Philadelphia.

POTAPOVA, M. \& D. F. CHARLES. 2003. Distribution of benthic diatoms in U.S. rivers in relation to conductivity and ionic composition. Freshwater Biol. 48: 1311-1328.

REICHARDT, E. 2008. Bemerkenswerte Diatomeenfunde aus Bayern VI. Hantzschia hyperborea (Grun.) Lange-B. Ber. Bayer. Bot. Ges. 78: 17-22.

RUMRICH, U. H., LANGE-BERTALOT \& M. RUMRICH. 2000. Iconographia Diatolmologica 9. Diatomeen der Anden von Venezuela bis Patagonien/ Tierra del Fuego. In: LANGE-BERTALOT, H. (ed.). A.R.G. Gantner Verlag K. G. Germany.

SALA, S. E., J. M. GUERRERO, M. COSTE, 1985. Valve morphology of Amphora chilensis Hustedt (Bacillariophyceae). Nova Hedwigia 85: 353-364.

SAR, E. A., S. E. SALA, I. SUNENSEN, M. S. HENNINGER, M. MONTASTRUC. 2009. Catálogo de los géneros, especies y taxa intraespecificos erigidos por J. Frenguelli. Diatom Monographs 10, A.R.G. Gantner Verlag K. G. Germany.

SCHMIDT, A., M. SCHMIDT, F. FRICKE, H. HEIDEN, O. MÜLLER \& F. HUSTEDT. 1874-1959. Atlas der 


\section{N. I. Maidana y C. T. Seeligmann - Diatomeas en humedales de Catamarca}

Diatomaceen-Kunde. R. Reisland, Leipzig.

SEELIGMANN C. \& N. I. MAIDANA. 2003. Diatomeas de la provincia de Catamarca (Argentina). Bol. Soc. Argent. Bot. 38: 39-50.

SEELIGMANN, C., N. I. MAIDANA, \& M. MORALES. 2008. Diatomeas (Bacillariophyceae) de Humedales de Altura de la provincia de Jujuy-Argentina. Bol. Soc. Argent. Bot. 43: 1-17.

SERVANT-VILDARY S. 1978. Les Diatomées des Sédiments superficiels d'un lac salé, Chloruré, sulfaté sodique de L'Altiplano Bolivien, le lac Poopó. ORSTOM ser. Geol. 1: 79-90.

SERVANT-VILDARY, S. 1984. Les Diatomées des lacs sursalés Boliviens. Sous classe Pennatphycidées, 1- Famille des Nitzschiacées. ORSTOM ser. Geol. 1: 35-52.

SERVANT-VILDARY, S., M. SERVANT \& O. JIMENEZ. 2001. Holocene hydrological and climatic changes in the southern Bolivian Altiplano according to diatom assemlages in paleowetlands. Hydrobiologia 466: 267-277.

SERVANT-VILDARY S., F. RISACHER \& M. ROUX. 2002. Diatom based transfer function for estimating the chemical composition of fossil water. Calibration based on salt lakes of the Lipez area in the southwestern Bolivian Altiplano. Carnets de Géologie / Notebooks on Geology, Memoir 2002/01: $1-11$.

SIMONSEN, R. 1987. Atlas and catalogue of the diatom types of Friedrich Hustedt. I-III. J. Cramer, Berlin.

TORGAN L. C. \& A. S. WEBER. 2008. Novos registros de Surirella Turpin (Bacillariophyta, Surirellaceae) para o Rio Grande do Sul e Brasil. Acta Bot. Bras. 22: 393-398.

VAN DAM, H., A. MERTENS \& J. SINKELDAM. 1994. A coded checklist and ecological indicator values of freshwater diatoms from the Netherlands. Netherlands J. Aq. Ecol. 28: 117-133.

VANLANDINGHAM, S. L. 1967-1979. Catalogue of the fossil and recent genera and species of diatoms and their synonyms. I-VIII. J. Cramer, Lehre.

VOUILlOUD, A. 2003. Catálogo de diatomeas continentales y marinas de Argentina. Versión 1.0. En soporte magnético. Asociación Argentina de Ficología, La Plata.

Recibido el 9 de marzo de 2015, aceptado el 27 de agosto de 2015 . 
Bol. Soc. Argent. Bot. 50 (4) 2015

\section{Apéndice. Especies identificadas en las muestras estudiadas.}

\begin{tabular}{|c|c|c|c|c|c|c|c|c|c|c|c|}
\hline Especies & 1 & 2 & 3 & 4 & 5 & 6 & 7 & 8 & 10 & 11 & 12 \\
\hline Achnanthes brevipes Agarth & & & & & & & & $\mathrm{X}$ & & & \\
\hline Achnanthidium exiguum (Grunow) Czarnecki & & & & & & & & & & & $\mathrm{x}$ \\
\hline Achnanthidium minutissimum (Kützing) Czarnecki & & & & & & & & & $\mathrm{X}$ & & $\mathrm{x}$ \\
\hline Achnanthidium thermalis var. rumrichorum Lange-Bertalot & & & & & & & & $\mathrm{x}$ & & & \\
\hline Adlafia minuscula (Grunow) Lange-Bertalot & & $\mathrm{X}$ & & & & $\mathrm{X}$ & & & & & \\
\hline Amphora affinis Kützing & & & & $\mathrm{X}$ & & & & & & & $\mathrm{x}$ \\
\hline Amphora copulata Schoemann \& Archibald & & & & & & $\mathrm{x}$ & & & & & \\
\hline Amphora lineolata Ehrenberg & & & & & & & & $\mathrm{X}$ & & $\mathrm{X}$ & \\
\hline Anomoeoneis punae sp nov. & & & & & & $x$ & & & & & $\mathrm{X}$ \\
\hline Anomoeoneis sphaerophora (Ehrenberg) & & & & & & & & & & & \\
\hline Pfitzer var. sphaerophora & & $\mathrm{x}$ & & & $\mathrm{x}$ & $x$ & & & & & \\
\hline Anomoeoneis sphaerophora fa. sculpta Krammer & $\mathrm{X}$ & & & & & & & & & & $\mathrm{x}$ \\
\hline $\begin{array}{l}\text { Anomoeoneis sphaerophora aff. var. } \\
\text { angusta Frenguelli Morf. } 1\end{array}$ & & & & & & $x$ & & & & & \\
\hline $\begin{array}{l}\text { Anomoeoneis sphaerophora aff. var. } \\
\text { angusta Frenguelli Morf. } 2\end{array}$ & & & & $\mathrm{X}$ & & & & & & & \\
\hline Anomoeoneis sphaerophora var. 1 & & & & & & & & & & & $\mathrm{x}$ \\
\hline Brachysira atacamae Hustedt & & & & & & & & $\mathrm{x}$ & & & \\
\hline Caloneis silicula (Ehrenberg) Cleve & & & & & & $x$ & & & $x$ & & \\
\hline Caloneis westii (W. Smith) Hendey & & & & & & & & $\mathrm{x}$ & & & $\mathrm{x}$ \\
\hline Cocconeis placentula var. euglypta (Ehrenberg) Grunow & & & & & & $x$ & & & & & $\mathrm{x}$ \\
\hline Cocconeis placentula var. lineata (Ehrenberg) Van Heurk & & & & & & $\mathrm{x}$ & & & & & \\
\hline Craticula accomoda (Hustedt) D.G.Mann & & & & & & & & & & & $x$ \\
\hline Craticula ambigua (Ehrenberg) D. G. Mann & & & & & & $\mathrm{X}$ & & & & & \\
\hline Cymbella cistula (Ehrenberg) Kirchner & & & & $\mathrm{x}$ & & $x$ & & & & & \\
\hline Cymbella cymbiformis Agardh & & & & & & $x$ & & & $\mathrm{X}$ & & $\mathrm{x}$ \\
\hline Denticula elegans Kützing & & $\mathrm{x}$ & & $\mathrm{x}$ & & $x$ & & & & & $x$ \\
\hline Denticula subtilis Grunow & & & & & & & & $\mathrm{x}$ & & & \\
\hline Denticula valida (Pedicino) Grunow & & & & $\mathrm{x}$ & & & $x$ & $\mathrm{x}$ & & & $\mathrm{x}$ \\
\hline Denticula aff. valida (Pedicino) Grunow & & & & $\mathrm{x}$ & & & & & & & \\
\hline Diploneis elliptica (Kützing) Cleve & & & $x$ & & & & & & & $\mathrm{x}$ & \\
\hline Discostella stelligera (Cl. \& Grun.) Houk \& Klee & & & $\mathrm{x}$ & & $\mathrm{x}$ & & & & & & \\
\hline Encyonema jemtlandicum var. venezolanum Krammer & & & & & & $x$ & & & & & \\
\hline Encyonopsis subfonticola Krammer & $\mathrm{X}$ & $\mathrm{X}$ & & & & & & & & & \\
\hline Encyonopsis aff. descripta var. asymmetrica Krammer & & & & & & $x$ & & & & & \\
\hline Encyonopsis aff. microcephala (Grunow) Krammer & & $\mathrm{X}$ & & & & & & & & & \\
\hline Eolimna minima Lange-Bertalot \& W. Schiller & & & & & & $x$ & & & & & \\
\hline Fallacia pygmaea Kützing & & & & & & & & $\mathrm{x}$ & & & $\mathrm{X}$ \\
\hline Gomphonema parvulum (Kützing) Kützing & & & & & & & & & & & $x$ \\
\hline Halamphora atacamae Frenguelli & & & & $x$ & & & & $\mathrm{x}$ & & $\mathrm{x}$ & \\
\hline Halamphora atacamana Patrick & $x$ & & & & & & & $x$ & & $x$ & $\mathrm{x}$ \\
\hline Halamphora carvajaliana (Patrick) Levkov & & & & & & & & $\mathrm{X}$ & & $\mathrm{X}$ & \\
\hline
\end{tabular}




\begin{tabular}{|c|c|c|c|c|c|c|c|c|c|c|c|}
\hline Especies & 1 & 2 & 3 & 4 & 5 & 6 & 7 & 8 & 10 & 11 & 12 \\
\hline Halamphora chilensis (Hustedt) Levkov & & & & & & & & $\mathrm{X}$ & & & \\
\hline Halamphora mira Krasske & & & & & & $\mathrm{X}$ & & $\mathrm{X}$ & & $\mathrm{X}$ & \\
\hline Halamphora oligotraphenta (Lange-Bertalot) Levkov & & & & & & & & $\mathrm{X}$ & & & \\
\hline Halamphora subsalina Levkov & & & & & & & & $\mathrm{X}$ & & & \\
\hline Halamphora tucumana (Herbst \& Maidana) Levkov & & & & & & $\mathrm{X}$ & & & & & \\
\hline Halamphora veneta Kützing & & & & $\mathrm{X}$ & & $\mathrm{X}$ & & & & & $\mathrm{X}$ \\
\hline Halamphora aff. Iucie (Cholnoky) Levkov & & & & & & & & $\mathrm{X}$ & & & \\
\hline Halamphora sp & & & & $\mathrm{X}$ & & & & & & & \\
\hline Haloraundia speciosa (Hustedt) Diaz \& Maidana & & & & & & & & $\mathrm{X}$ & & & \\
\hline Hantzschia hyperborea (Grunow) Lange-Bertalot & & & & & & $x$ & & & & & \\
\hline Hyalodiscus lentiginosus John & & & $\mathrm{X}$ & & & & & & & & \\
\hline Luticola mollis Lange-Bertalot \& Rumrich & & & & & & $x$ & & & $\mathrm{X}$ & & \\
\hline Luticola muticopsis (Van Heurck) D. G. Mann & & & & $\mathrm{X}$ & & $\mathrm{X}$ & & & & & \\
\hline Mastogloia atacamae Hustedt & & & & & & $\mathrm{X}$ & & & & & \\
\hline Mastogloia elliptica Agardh & & & & $\mathrm{X}$ & & & $\mathrm{X}$ & $\mathrm{X}$ & & $\mathrm{X}$ & $\mathrm{x}$ \\
\hline Mastogloia aff. patens Frenguelli & & & & & & & & $\mathrm{X}$ & & & \\
\hline Microcostatus andinus & & & & & $\mathrm{X}$ & & & & & $x$ & \\
\hline Muelleria aff. patagonica (O. Müller) Frenguelli & & & & & & & & & $\mathrm{x}$ & & \\
\hline Navicella pusilla (Grunow) Krammer & & & & & & $\mathrm{X}$ & & $\mathrm{X}$ & & & \\
\hline Navicula atacamana Patrick & & & & & & & & $\mathrm{X}$ & & & $\mathrm{X}$ \\
\hline Navicula cari Ehrenberg & $\mathrm{x}$ & & & & & & & & & & \\
\hline Navicula cincta (Ehrenberg) Ralfs & & & & & & $\mathrm{x}$ & $\mathrm{x}$ & & $\mathrm{x}$ & & $\mathrm{x}$ \\
\hline Navicula cryptotenella Lange-Bertalot & & & & & & & & $\mathrm{X}$ & & & \\
\hline Navicula cryptotenelloides Lange-Bertalot & & & & & $\mathrm{X}$ & & $\mathrm{X}$ & & & & \\
\hline Navicula digitoradiata var. minor Krasske & & $X$ & & & & & & & & & \\
\hline Navicula lauca Rumrich \& Lange-Bertalot & $\mathrm{X}$ & $\mathrm{X}$ & & $\mathrm{X}$ & & $\mathrm{X}$ & & $\mathrm{X}$ & $\mathrm{X}$ & & $\mathrm{X}$ \\
\hline Navicula libonensis Schoemann & & $\mathrm{X}$ & & & & & & & & & \\
\hline Navicula microdigitoradiata Lange- Bertalot & & & & & & & $\mathrm{X}$ & $\mathrm{X}$ & $\mathrm{X}$ & $x$ & \\
\hline Navicula parinacota Rumrich \& Lange-Bertalot & $\mathrm{X}$ & $X$ & $x$ & $X$ & & & $\mathrm{X}$ & $\mathrm{X}$ & & $\mathrm{X}$ & $\mathrm{x}$ \\
\hline Navicula pseudogracilis Hustedt & $\mathrm{x}$ & & & & & $\mathrm{x}$ & $\mathrm{x}$ & & & $\mathrm{X}$ & $\mathrm{x}$ \\
\hline Navicula salinicola Hustedt var. salinicola & & $\mathrm{X}$ & & $\mathrm{X}$ & $\mathrm{X}$ & & & & & $\mathrm{X}$ & \\
\hline Navicula salinicola var. boliviana Patrick & & $\mathrm{X}$ & & & $X$ & & $\mathrm{x}$ & $\mathrm{x}$ & & $\mathrm{X}$ & \\
\hline Navicula veneta Kützing & $\mathrm{X}$ & & & & $\mathrm{x}$ & & & & & $\mathrm{x}$ & $\mathrm{x}$ \\
\hline Navicula aff. cincta Ehrenberg & & & & $\mathrm{X}$ & & & & & & & \\
\hline Navicula aff. microdigitoradiata Lange-Bertalot & & & & & & & $\mathrm{x}$ & & & & \\
\hline Navicula aff. pseudogracilis Hustedt & & & & & & & & $\mathrm{X}$ & & & \\
\hline Navicula sp 1 & & & & & & & & & & & $\mathrm{X}$ \\
\hline Navicula sp 2 & & & & & & & & & & $x$ & \\
\hline Naviculadicta chilensis (Krasske) Lange-Bertalot & & & & & & & & $\mathrm{X}$ & & & \\
\hline Neidium ampliatum (Ehrenberg) Krammer & & & $\mathrm{x}$ & & & & & & & & \\
\hline Neidium bisulcatum var. subampliatum Krammer & & & & & & & & & $\mathrm{X}$ & & \\
\hline Nitzschia capitellata Hustedt & & & & & & & & & $x$ & & \\
\hline
\end{tabular}


Bol. Soc. Argent. Bot. 50 (4) 2015

\begin{tabular}{|c|c|c|c|c|c|c|c|c|c|c|c|}
\hline Especies & 1 & 2 & 3 & 4 & 5 & 6 & 7 & 8 & 10 & 11 & 12 \\
\hline Nitzschia conmutata Grunow & & & & & & & & $\mathrm{x}$ & & & $\mathrm{x}$ \\
\hline Nitzschia epithemioides Grunow & & & $\mathrm{x}$ & & $\mathrm{x}$ & & & $\mathrm{x}$ & & $\mathrm{x}$ & \\
\hline Nitzschia gracilis Hantsch & & $\mathrm{x}$ & & & & $\mathrm{x}$ & & $x$ & & & \\
\hline Nitzschia halloyii Maidana \& Herbst & & $\mathrm{x}$ & & $\mathrm{x}$ & & $\mathrm{x}$ & & $x$ & $\mathrm{x}$ & $\mathrm{x}$ & $\mathrm{x}$ \\
\hline Nitzschia inconspicua Grunow & & $\mathrm{x}$ & & & & $x$ & & & $\mathrm{x}$ & & \\
\hline Nitzschia liebetruthii Rabenhorst & & & & & $x$ & & & & & & \\
\hline Nitzschia palea (Kützing) W. Smith & & & & & & & & & $\mathrm{x}$ & & \\
\hline Nitzschia pusilla Grunow & $\mathrm{x}$ & & $\mathrm{x}$ & & $\mathrm{x}$ & & & $\mathrm{x}$ & & & $\mathrm{x}$ \\
\hline Nitzschia subacicularis Hustedt & & & & & & & & & & & $x$ \\
\hline Nitzschia valdecostata L.-Bertalot \& Simonsen & & $\mathrm{x}$ & & $\mathrm{x}$ & & & & $\mathrm{x}$ & & & $x$ \\
\hline Nitzschia valdestriata Aleem \& Hustedt & & & & $\mathrm{x}$ & & & & $\mathrm{x}$ & & & \\
\hline Nitzschia vitrea G. Norman & & & & & & $x$ & & & & & \\
\hline Pinnularia maior (Kützing) Rabenhorts & & & & & & & & & & $\mathrm{x}$ & \\
\hline Pinnularia microstauron (Ehrenberg) Cleve & $\mathrm{x}$ & $\mathrm{x}$ & & $\mathrm{x}$ & & $\mathrm{x}$ & $\mathrm{x}$ & $\mathrm{x}$ & $\mathrm{x}$ & & $\mathrm{x}$ \\
\hline Pinnularia aff. boliviana Blanco et al. & & $\mathrm{x}$ & & & & & & & & & \\
\hline Placoneis australis Zidarova et al. & & & & & & $\mathrm{x}$ & & & $\mathrm{x}$ & & \\
\hline Planothidium chilense (Hustedt) L.-Bertalot & & & & & & & & $\mathrm{x}$ & & & \\
\hline Planothidium delicatulum (Kützing) Round \& Bukhtiyarova & & & & & & & & $\mathrm{x}$ & & $\mathrm{x}$ & $\mathrm{x}$ \\
\hline Planothidium dubium (Grunow) Round \& Bukhtiyarova & & & & & & & & $\mathrm{x}$ & & & \\
\hline Planothidium frequentissimum (L.- & & & & & & & & & & & \\
\hline Bertalot) Round \& Bukhtiyarova & & & & & & $x$ & & & & & $x$ \\
\hline Planothidium lanceolatum (Brébisson) Round \& Bukhtiyarova & & & & & & $x$ & & & & & $x$ \\
\hline Planothidium aff. minutissimum (Mayer) Morales & & & & & & & & $\mathrm{x}$ & & & $x$ \\
\hline Proschkinia bulnheimii (Grunow) Karayeva & & & & $\mathrm{X}$ & & & & $x$ & & $x$ & \\
\hline Rhopalodia constricta (W. Smith) Krammer & & & & & & & & $x$ & & & \\
\hline Rhopalodia gibberula (Ehrenberg) O. Müller & & & & & & & $x$ & & & & \\
\hline Rhopalodia aff. wetzelii Hustedt & & & & & & & $x$ & $\mathrm{x}$ & & & \\
\hline Scoliopleura peisonis Grunow & & & $x$ & $x$ & & & & & $\mathrm{x}$ & $\mathrm{x}$ & \\
\hline Sellaphora pupula (Kützing) Mereschkowksy & & & & & & $\mathrm{x}$ & & & & & \\
\hline Staurophora aff. Navicula carvajaliana Patrick & & $x$ & & & $\mathrm{x}$ & & $\mathrm{x}$ & & & $\mathrm{x}$ & \\
\hline Staurophora aff. Stauroneis atacamae Hustedt & & & & & & $\mathrm{x}$ & & & & & \\
\hline Staurophora sp 1 aff. Navicula luissi Patrick & & & & & & & $x$ & & & & \\
\hline Staurophora vilamae Maidana \& seeligmann & & & & & & & $x$ & $x$ & $\mathrm{x}$ & & \\
\hline Surirella chilensis Janish & $\mathrm{x}$ & & $x$ & $x$ & & $\mathrm{x}$ & & $\mathrm{x}$ & & $\mathrm{x}$ & $x$ \\
\hline Surirella chilensis aff. var. tumida Hustedt & & & $x$ & $x$ & & & & & & & $x$ \\
\hline Surirella forti Frenguelli & $\mathrm{x}$ & & & $\mathrm{X}$ & & & & & & & \\
\hline Surirella moralesi Blanco & & & & & & & & & & & $x$ \\
\hline Surirella sella Hustedt & & & & & & & & $\mathrm{x}$ & & & \\
\hline Surirella tuberosa Frenguelli & $\mathrm{x}$ & & & & & & & & & & \\
\hline Surirella utahensis Salah & $x$ & & & & & $\mathrm{x}$ & & & & & \\
\hline Surirella wetzelii Hustedt & & & $\mathrm{X}$ & & $\mathrm{X}$ & & $\mathrm{x}$ & & & $\mathrm{x}$ & \\
\hline Tryblionella hungarica (Grunow) Frenguelli & $\mathrm{x}$ & & & $\mathrm{X}$ & & $x$ & & $x$ & $x$ & $x$ & \\
\hline Ulnaria ulna (Nitzsch) Compère & & & & & & & & & & & $x$ \\
\hline TOTAL DE ESPECIES & 15 & 18 & 11 & 25 & 12 & 39 & 16 & 47 & 18 & 25 & 38 \\
\hline
\end{tabular}

